\title{
Métodos utilizados na Ergonomia Organizacional: revisão de literatura
}

\section{Methods used in Organizational ergonomics: Literature Review}

\author{
Alais Souza Ferreira ${ }^{1}$ \\ Eugenio Andrés Díaz Merino ${ }^{2}$ \\ Luiz Fernando Gonçalves de Figueiredo ${ }^{3}$
}




\section{Resumo}

O artigo objetiva conceituar a ergonomia organizacional e identificar os métodos e técnicas utilizados, preferencialmente no contexto dos métodos de pesquisa ergonômica (MPE's). A pesquisa é classificada como básica, teórica, qualitativa e exploratória, composta pela revisão de literatura tradicional. A população de artigos científicos é constituída por 544 e a amostra por 20 (apresentados em um quadro). Ressalta-se: que o mapeamento por entrevista é o método mais utilizado, seguido pelo mapeamento por questionário, grupo de foco e ergonomia participativa; e existe uma variedade de métodos aplicados na ergonomia organizacional que podem ser combinados.

Palavras-chave: ergonomia; ergonomia organizacional; métodos de pesquisa ergonômica; métodos organizacionais.

\section{Abstract}

The article aims to conceptualize organizational ergonomics and identify the methods and techniques used, preferably in the context of the ergonomic research methods (ERM's). The research is classified as basic, theoretical, qualitative and exploratory, composed of the revision of traditional literature. The population of scientific articles consists of 544 and the sample by 20 (presented in a framework). It is emphasized: that the mapping by interview is the most used method, followed by the mapping by questionnaire, focus group and participatory ergonomics; And there is a variety of methods applied in organizational ergonomics that can be combined.

Key-words: Ergonomics; Organizational ergonomics; Ergonomic research methods; Organizational methods.

ISSN: 2316-7963

${ }^{1}$ Bacharel em Design Gráfico (alais.ferreira@live.com)

2 Doutor em Engenharia, UFSC

${ }^{3}$ Doutor em Engenharia, UFSC 


\section{Introdução}

A ergonomia é a ciência do trabalho que estuda as intervenções entre os seres humanos e outros elementos do sistema, aplicando teorias, princípios, dados e métodos a projetos que busquem otimizar o bem estar humano e o desempenho global de sistemas. Seu objetivo é a satisfação e conforto dos indivíduos, a garantia que a prática laboral e o uso de equipamentos não causem problemas a saúde do usuário. A discussão sobre ergonomia começou por volta de 1857 (MÁSCULO; VIDAL, 2011) e passou por diversos períodos: o clássico, ocorrido na primeira metade do século $\mathrm{XX}$; segunda guerra mundial em que a importância estava nos fatores humanos do pós-guerra; até chegar ao Brasil. Mais especificamente, de acordo com pesquisas publicadas, a ergonomia nasceu em 12 de julho de 1949, após a II Guerra Mundial, como consequência do trabalho interdisciplinar realizado por diversos profissionais (engenheiros, fisiologistas e psicólogos) durante a guerra (IEA, 2000; IIDA, 2005; HENDRICK; KLEINER, 2006; ABRAHÃO et al., 2009; MÁSCULO; VIDAL, 2011; MERINO, 2011).

Esta ciência possui três domínios de especialização: física, cognitiva e organizacional. $O$ foco deste artigo está na ergonomia organizacional que é a otimização dos sistemas sociotécnicos, com a inclusão das estruturas organizacionais, políticas e processos. Esta não se constitui em sistema fechado, já que exige contínuo desenvolvimento, aplicação e validação de dados. A ergonomia organizacional possui como sistemas sociotécnicos o subsistema tecnológico, o subsistema pessoal, o ambiente externo, e o projeto organizacional, que interagem entre si e podem ser utilizados para determinar a estrutura ótima do sistema de trabalho.

Tanto a ergonomia como a ergonomia organizacional precisam ter um conjunto de conhecimentos, métodos e práticas que cada interventor pode mobilizar em relação à especificidade de suas intervenções. Neste contexto, Maggi $(2006$, p.16) informa que a reflexão epistemológica das ciências humanas e sociais aumentou com o debate sobre os métodos, chamado de "Methodenstrei". Este se desenvolveu no âmbito do historicismo alemão entre as duas últimas décadas do século XIX e a primeira do século XX, pois houve um confronto entre "aqueles que defendem a unidade do método científico contra aqueles que defendem a especificidade das ciências do espírito, ou ciências da cultura". O autor continua relatando que o filósofo e historiador alemão Wilheim Dilthey, expõe que as ciências metodológicas estudam um campo do qual o ser humano é uma parte integrante e do qual ele tem um conhecimento que vem de sua experiência. Dessa forma, os métodos contribuem para tentar reviver a experiência subjetiva de outros sujeitos e compreender o sentido de suas ações.

Inserido neste cenário, está à ergonomia e suas especialidades que são ciências experimentais, semelhantes a ciências sociais, uma vez que constroem os seus conhecimentos a partir de observações e experimentações, em condições controladas e comprovadas, pela mensuração dos fenômenos. Hendrick e Kleiner (2006) informam que os métodos da ergonomia organizacional não se constituem num conjunto fechado, pois eles têm sido continuamente desenvolvidos, aplicados e validados.

Com isso, surge o questionamento: Quais são os métodos de ergonomia organizacional existentes? E para quais finalidades estes têm sido utilizados? O artigo objetiva identificar os métodos e técnicas utilizadas, preferencialmente no contexto de mét- 
odos de pesquisa ergonômica (MPE's) organizacional. Para alcançá-lo é necessário: compreender a ergonomia e seus aspectos organizacionais, mediante um levantamento bibliográfico; conceituar ergonomia e ergonomia organizacional; e identificar os métodos de ergonomia organizacional nos artigos científicos coletados por meio da revisão de literatura.

O artigo é caracterizado por sua natureza como pesquisa básica e teórica, pela abordagem como qualitativo, pelos objetivos como exploratório (GIL, 2010; VIRGILLITO, 2010; CRESWELL, 2016). De acordo com os procedimentos metodológicos a pesquisa é classificada como revisão de literatura tradicional, realizada em junho de 2017, e levantamento bibliográfico de dados secundários (GIL, 2010; VIRGILLITO, 2010; CRESWELL, 2016), por utilizar pesquisas publicadas em bases de dados eletrônicos e livros.

Este trabalho justifica-se, pois de acordo com pesquisas publicadas por Hendrick (1995) e lida (2005) a ergonomia organizacional ou macroergonomia permite que muitas decisões ergonômicas sejam tomadas em nível da administração superior da empresa. Isso contribui na melhoria da segurança, satisfação, saúde e produtividade, além de reduzir erros e acidentes em toda a empresa. Os autores finalizam informando que essa visão macroergonômica tem proporcionado, em alguns casos, resultados mais satisfatórios do que a abordagem microergonômica dos trabalhadores individuais ou em postos de trabalho isolados. Dessa forma, a abordagem micro pode produzir melhorias de 10 a $25 \%$ enquanto a abordagem macro pode proporcionar melhorias de 60 a $90 \%$.

O artigo está estruturado em sete seções. A primeira é constituída por uma introdução sobre ergonomia, ergonomia organizacional, questões, objetivos e metodologia da pesquisa. A segunda refere-se à ergonomia, relatando o seu conceito, objetivo e suas especialidades. A terceira aborda a ergonomia organizacional informando o conceito, objetivo e os MPE's. A quarta expõe as etapas metodológicas desenvolvidas, bem como esta pesquisa está classificada. E a quinta apresenta uma discussão teórica sobre os métodos de ergonomia organizacional encontrados nos materiais coletados. A sexta seção apresenta uma conclusão das informações relatadas nesta pesquisa.

\section{Ergonomia}

Esta seção relata quando surgiu à ergonomia, o significado do termo, a definição do conceito e suas especialidades. De acordo com pesquisas publicadas, o nascimento da ergonomia ocorreu em 12 de julho de 1949, quando um grupo de cientistas e pesquisadores reuniu-se na Inglaterra para discutir e formalizar a existência desse novo ramo de aplicação interdisciplinar da ciência. Na segunda reunião realizada por esse mesmo grupo, ocorrida em 16 de fevereiro de 1950, foi proposto o termo ergonomia formado pelas palavras gregas "ergon" que significa trabalho e "nomos" que significa regras (MURREL, 1965; IIDA, 2005; MERINO, 2011).

Dentre as diversas definições do conceito de ergonomia estabelecidas pelas instituições Ergonomics Society (Inglaterra), Associação Brasileira de Ergonomia (Brasil), International Ergonomics Association (âmbito internacional), Société d'ergonomie de langue française (França) destaca-se que todas visam ressaltar o caráter interdisciplin- 
ar e a interação entre o homem e o trabalho no sistema homem-máquina-ambiente (IIDA, 2005; FALZON, 2007). O artigo está embasado na definição da International Ergonomics Association (IEA, 2000) que considera a ergonomia: uma disciplina científica que estuda as intervenções entre os seres humanos e outros elementos do sistema; e a profissão que aplica teorias, princípios, dados e métodos a projetos que busquem otimizar o bem estar humano e o desempenho global de sistemas (MURREL, 1965; WISNER, 1987; GUÉRIN et al., 2001; IIDA, 2005; HENDRICK e KLEINER, 2006; ABRAHÃO et al., 2009; MÁSCULO e VIDAL, 2011; MERINO, 2011).

Portanto, a ergonomia é a ciência do trabalho e está orientada para uma abordagem sistêmica de todos os aspectos da atividade humana. Dessa forma, esta disciplina busca analisar a adequação do trabalho e do ambiente em que as atividades são executadas ao ser humano. Seu objetivo é estudar os diversos fatores que interferem no desempenho do sistema produtivo, reduzir as suas consequências nocivas sobre o trabalhador (como fadiga, estresse, erros e acidentes), com o intuito de proporcionar mais segurança, satisfação e saúde aos trabalhadores durante sua interação com o sistema produtivo. (IIDA, 2005; FALZON, 2007; MERINO, 2011; CORRÊA e BOLETTI, 2015). A ergonomia inicia-se com o estudo das características do trabalhador para então projetar o trabalho que ele consegue executar respeitando suas capacidades e limitações. Esse estudo envolve não apenas o ambiente físico, mas também os aspectos organizacionais e cognitivos.

Inicialmente a ergonomia era aplicada exclusivamente na indústria e se concentrava apenas na interação constituída pelo binômio homem-máquina. Com o passar dos anos, a ergonomia se tornou mais abrangente, estudando sistemas complexos, onde dezenas ou até centenas de homens, máquinas e materiais interagem continuamente entre si, na realização de um trabalho (IIDA, 2005). Dessa forma esta ciência se expandiu horizontalmente, envolvendo quase todos os tipos de atividades humanas.

A ergonomia se constituiu a partir do projeto de construir dois tipos de conhecimentos: o primeiro sobre o homem por meio de uma abordagem holística, onde este é pensado simultaneamente em suas dimensões fisiológicas, cognitivas e sociais, produzindo conhecimentos úteis à ação, quer se trate da transformação ou da concepção de situações de trabalho ou objetos técnicos; e por outro lado, os conhecimentos sobre a ação ergonômica envolvendo metodologias de análise e intervenção nas situações de trabalho, metodologias de participação na concepção, e avaliação dos dispositivos técnicos e organizacionais (FALZON, 2007). Com isso, a ergonomia possui dois objetivos: o primeiro centrado nas organizações e no seu desempenho considerando os aspectos de eficiência, produtividade, confiabilidade, qualidade, durabilidade etc; já o segundo está centrado nas pessoas e desdobra-se em diferentes dimensões como a segurança, saúde, conforto, facilidade de uso, satisfação, interesse do trabalho, prazer etc.

Inserido neste contexto, estão os profissionais que praticam a ergonomia denominados como ergonomistas. Estes "realizam o planejamento, projeto e avaliação de tarefas, postos de trabalho, produtos, ambientes e sistemas, tornando-os compatíveis com as necessidades, habilidades e limitações das pessoas", ou seja, precisam analisar o trabalho de forma global incluindo os aspectos físicos, cognitivos, sociais, organizacionais e ambientais. (IIDA, 2005, p. 3; FALZON, 2007). Esses profissionais trabalham 
em domínios especializados, tais como: ergonomia física, cognitiva e organizacional. O foco deste artigo está na ergonomia organizacional que será apresentada na próxima seção.

\section{Ergonomia Organizacional}

Aqui será abordada a definição de ergonomia organizacional, quando a mesma surgiu, os tipos de conhecimentos até chegar ao assunto sobre os MPE's. A área de atuação da ergonomia ampliou-se a partir da década de 1980 denominada como ergonomia organizacional, ou macroergonomia, que é considerada uma subdisciplina. Nesta nova visão a ergonomia é definida como o desenvolvimento e aplicação da tecnologia da interface homem-máquina em toda a organização, passando a fazer parte do projeto e gerência de organizações (HENDRICK; KLEINER, 2006).

A ergonomia organizacional visa à otimização dos sistemas sócio-técnicos, incluindo as estruturas organizacionais, políticas ou regras, e processos. Esta especialização da ergonomia compreende alguns temas relevantes como: as comunicações; a concepção do trabalho; a gestão dos coletivos; a concepção dos horários de trabalho; programação do trabalho em grupo; ergonomia comunitária; as novas formas de trabalho; projeto participativo; trabalho cooperativo; cultura organizacional; organizações em rede; teletrabalho; e gestão da qualidade (IIDA, 2005; HENDRICK; KLEINER, 2006; FALZON, 2007; MÁSCULO; VIDAL, 2011).

Segundo Hendrick e Kleiner (2006) esta ciência possui como sistemas sociotécnicos: o subsistema tecnológico; o subsistema pessoal; o ambiente externo; e o projeto organizacional. Esses sistemas interagem entre si de modo que uma mudança em qualquer um deles afeta os outros três (geralmente na forma de desfuncionamento ou imprevisto), e podem ser utilizados para determinar a estrutura ótima do sistema de trabalho. Ao desenhar estes sistemas, a dimensão macroergonômica acaba determinando muitos aspectos do desenho microergonômico do sistema de trabalho e isso garante a compatibilidade ergonômica dos componentes de sistemas com a estrutura global do sistema de trabalho. Em relação aos sistemas sóciotécnicos, esta subdisciplina otimiza de forma integrada os subsistemas técnicos e de pessoal de toda a organização, além de produzir a harmonização dos elementos do sistema de trabalho com seu desenho global e o ambiente externo. Segundo Hendrick e Kleiner (2006, p. 49), isto tudo resulta em maior garantia de um ótimo funcionamento e efetividade do sistema, incluindo "os aspectos de produtividade, qualidade, saúde e segurança do empregado, fatores psicossociais de conforto, motivação intrínseca, compromisso e percepção da qualidade de vida no trabalho".

A macroergonomia é uma abordagem descendente, pois esta precisa assegurar que o projeto: geral do sistema de trabalho seja compatível com as características do sistema sociotécnico da organização; e o das subunidades e componentes do sistema de trabalho se harmonizem com o projeto geral (HENDRICK; KLEINER, 2006). O autor ainda informa que o processo de projeto da macroergonomia, ou seja, as estruturas e processos que constituem o sistema geral de trabalho podem ser analisados e desenhados de três formas, começando com: a estrutura e os processos gerais de sistema de trabalho, trabalhando para baixo através dos subsistemas e dos componentes do sistema (descendente ou top-down); os componentes, e sistematicamente construindo as estruturas e processos do sistema geral de trabalho (ascendente ou bottom-up); 
ou em um nível intermediário na organização, e sistematicamente construindo ambos em cima e em baixo (radial ou middle-out).

Os objetivos da psicologia organizacional também são importantes para a macroergonomia, são eles: encorajar equipes de trabalho; melhorar a liderança e a motivação; a satisfação da tarefa; e implantar sistemas de incentivo. Porém, o foco primário da macroergonomia é

desenhar sistemas de trabalho compatíveis com as características do sistema sociotécnico da organização e então transferir aquele projeto de sistema de trabalho através do projeto de interfaces de humano-tarefa, humano-máquina, humano-programa e humano-ambiente para assegurar um sistema de trabalho totalmente harmonizado (HENDRICK; KLEINER, 2006, p. 20).

Tanto a ergonomia como a macroergonomia são ciências experimentais, semelhantes as naturais e sociais, pois constroem seus conhecimentos a partir de observações e experimentações, em condições controladas e comprovadas, pela mensuração dos fenômenos (IIDA, 2005; STANTON et al., 2005; FALZON, 2007). Por exemplo, faz observações reais da influência do clima no trabalho. Para isso, essas disciplinas utilizam métodos de pesquisa ergonômica (MPE's) que são procedimentos ou caminhos utilizados pelo pesquisador para estabelecer a relação entre causa e efeito, composto por várias etapas, partindo de uma hipótese para se chegar ao resultado da pesquisa, confirmando ou rejeitando essa hipótese. Hendrick e Kleiner (2006, p. 53) relatam que "os métodos da macroergonomia não se constituem num conjunto fechado, eles têm sido continuamente desenvolvidos, aplicados e validados". Já as técnicas "são operações, ações ou modos de executar uma atividade", por isso o método é mais amplo que a técnica e pode ser composto por várias técnicas. "Para cada método existe um conjunto de técnicas adequadas, para se chegar ao fim pretendido. Por sua vez, a mesma técnica pode ser usada em vários tipos de métodos" (IIDA, 2005, p. 35).

A escolha do método e das técnicas depende muito do objetivo pretendido, dos recursos e tempos disponíveis, da habilidade e experiência do pesquisador, e das restrições que ele encontra na prática. "Em princípio, não se pode afirmar que haja um método adequado para cada tipo de problema, devido a essas condições limitantes" (IIDA, 2005, p. 36). Dentre os tipos de métodos possuem os participativos, que são aqueles em que o pesquisador deixa de ser observador e passa a fazer parte ativa da solução do problema, e os convencionais, onde o pesquisador não interfere e não participa da situação observada. Além disso, nos métodos participativos pode haver a inclusão dos usuários ou consumidores na solução do problema, lembrando que nos convencionais os usuários não são considerados atores relevantes na busca da solução e sim meros informantes e executores das ações.

Segundo lida (2005), inicialmente a aplicação da ergonomia com suas especialidades se restringia à indústria, ao setor militar e aero-espacial. Com o passar dos anos, a aplicação se expandiu para a agricultura, setor de serviços e à vida diária do cidadão comum. Porém, esse alargamento exigiu novos conhecimentos, como as características de trabalho de mulheres, pessoas idosas e aqueles portadores de deficiências físicas. O quadro 1 sintetiza os MPE's, mencionados pelos autores citados, e onde podem ser aplicados na ergonomia organizacional. 


\section{Quadro 1. Métodos encontrados nos livros}

\begin{tabular}{|c|c|c|}
\hline Autor: & Método:A & plicação: \\
\hline $\begin{array}{l}\text { Wilson (1995), } \\
\text { lida (2005), } \\
\text { Hendrick e } \\
\text { Kleiner (2006) }\end{array}$ & $\begin{array}{l}\text { Ergonomia } \\
\text { participativa }\end{array}$ & $\begin{array}{l}\text { Pode ser aplicada na participação: de tomada de decisão } \\
\text { e em resolução de problema; em produto e desenho do } \\
\text { sistema; no projeto de treinamentos; e em análise e } \\
\text { projeto de sistemas de trabalho. }\end{array}$ \\
\hline $\begin{array}{l}\text { lida (2005) e } \\
\text { Thiollent (2011) }\end{array}$ & Pesquisa-ação & Utilizada na resolução de problemas. \\
\hline \multirow[t]{2}{*}{$\begin{array}{l}\text { Hendrick e } \\
\text { Kleiner (2006) }\end{array}$} & Estudo de campoN & $\begin{array}{l}\text { a análise dos registros de desempenho existentes para } \\
\text { um determinado sistema de trabalho, e no estudo das } \\
\text { características deste sistema e das características }\end{array}$ \\
\hline & & $\begin{array}{l}\text { sociotécnicas da organização. Isso permite identificar } \\
\text { áreas de problemas que são possíveis de intervenções } \\
\text { macroergonômicas e aumentar a visão da natureza e } \\
\text { escopo da intervenção. }\end{array}$ \\
\hline \multirow[t]{5}{*}{$\begin{array}{l}\text { lida }(2005) \\
\text { Hendrick e } \\
\text { Kleiner (2006) }\end{array}$} & $\begin{array}{l}\text { Experiência de } \\
\text { laboratório }\end{array}$ & $\begin{array}{l}\text { Este método é utilizado para manipular sistematicamente } \\
\text { as variáveis do sistema de trabalho e as sociotécnicas de } \\
\text { interesse (como a complexidade, centralização e } \\
\text { formalização), e então observar e registrar } \\
\text { sistematicamente o impacto destas no desempenho de } \\
\text { outras variáveis de interesse. }\end{array}$ \\
\hline & $\begin{array}{l}\text { Experiência de } \\
\text { campo }\end{array}$ & $\begin{array}{l}\text { Pode ser usada como um prosseguimento de um estudo } \\
\text { de campo inicial. Por vez, isto poderá ser feito em uma } \\
\text { parte particular da organização para testar sua utilidade e } \\
\text { então, no caso de se provar algo efetivo, ser } \\
\text { implementado em uma escala maior na empresa. } \\
\text { Geralmente é combinado com a ergonomia participativa. }\end{array}$ \\
\hline & $\begin{array}{l}\text { Mapeamento por } \\
\text { questionário } \\
\text { organizacional }\end{array}$ & $\begin{array}{l}\text { Utilizado para identificar sintomas de problemas no projeto } \\
\text { do sistema de trabalho - de forma rápida e de baixo custo } \\
\text { - e ainda localizando onde podem estar ocorrendo esses } \\
\text { problemas. Quando usado em sua forma de realimentação } \\
\text { de pesquisa como uma parte integrante do processo de } \\
\text { ergonomia participativa, pode proporcionar para gerentes } \\
\text { e empregados uma série de dados que lhes ajudem a } \\
\text { identificar problemas no projeto do sistema de trabalho } \\
\text { e/ou sugerir o que talvez precise ser feito para corrigir ou } \\
\text { melhorar o funcionamento. Pode ser usado também como } \\
\text { um instrumento de medida comparando a situação } \\
\text { antecedente e a situação posterior à ação } \\
\text { macroergonômica, apontando oportunidades de } \\
\text { continuidade da intervenção e do redesenho do sistema } \\
\text { de trabalho. }\end{array}$ \\
\hline & $\begin{array}{l}\text { Mapeamento por } \\
\text { entrevista }\end{array}$ & $\begin{array}{l}\text { Entrevistas semiestruturadas são utilizadas para: } \\
\text { identificar e obter uma visão dos problemas com o atual } \\
\text { desenho de um sistema de trabalho; apontar que tipos } \\
\text { específicos de intervenção macroergonômica poderiam } \\
\text { ser efetivos, seja redesenhando o sistema de trabalho ou } \\
\text { implementando o redesenho; identificar incompatibilidades } \\
\text { entre o macro projeto do sistema de trabalho e o micro- } \\
\text { desenho de ocupações individuais, e os subsistemas } \\
\text { humano-máquina e de interfaces de humano-software. }\end{array}$ \\
\hline & Grupo de foco & $\begin{array}{l}\text { Quando aplicado dentro do contexto macroergonômico, é } \\
\text { utilizado para trazer as pessoas de um sistema de trabalho } \\
\text { particular para serem entrevistadas sobre aspectos } \\
\text { específicos desse sistema ou de seu ambiente } \\
\text { sociotécnico. Pode ser conduzido como uma entrevista } \\
\text { semiestruturada. Em alguns casos, é feita uma simulação } \\
\text { de mudanças no sistema de trabalho entrevistando os } \\
\text { interesses desses grupos em conjunto para obter suas } \\
\text { percepções coletivas ou suas opiniões sobre aspectos } \\
\text { específicos da mudança. }\end{array}$ \\
\hline
\end{tabular}

(continua) 


\begin{tabular}{|c|c|c|}
\hline \multirow[t]{3}{*}{$\begin{array}{l}\text { Stanton et al. } \\
(2005)\end{array}$} & $\begin{array}{l}\text { Cognitive Walk- } \\
\text { Through Method } \\
\text { (CWM) }\end{array}$ & Problemas de interface de um produto. \\
\hline & Kansei Engineering & $\begin{array}{l}\text { Traduzir as respostas afetivas dos consumidores em } \\
\text { novos produtos ergonômicos. }\end{array}$ \\
\hline & HITOP Analysis & Implementar mudanças tecnológicas. \\
\hline & TOP-Modeler & $\begin{array}{l}\text { Sistema de apoio à decisão para identificar as mudanças } \\
\text { organizacionais necessárias quando novas tecnologias de } \\
\text { processo estão sendo consideradas. }\end{array}$ \\
\hline & CIMOP System & $\begin{array}{l}\text { Sistema de base de conhecimento para avaliar a } \\
\text { fabricação, organização e projeto de sistema. }\end{array}$ \\
\hline & Anthropotechnology & $\begin{array}{l}\text { Trata especificamente da análise e da modificação dos } \\
\text { sistemas de transferência de tecnologia de uma cultura } \\
\text { para outra. }\end{array}$ \\
\hline & $\begin{array}{l}\text { Systems Analysis } \\
\text { Tool (SAT) }\end{array}$ & $\begin{array}{l}\text { Adaptação, elaboração e etapas básicas do método } \\
\text { científico. }\end{array}$ \\
\hline & $\begin{array}{l}\text { Macroergonomic } \\
\text { Analysis of Structure } \\
\text { (MAS) }\end{array}$ & $\begin{array}{l}\text { Avaliar a estrutura dos sistemas pelos aspectos: } \\
\text { tecnologia do sistema de trabalho, subsistema de pessoal } \\
\text { e ambiente externo ao qual a organização deve responder } \\
\text { para sobreviver e ser bem sucedida. }\end{array}$ \\
\hline & $\begin{array}{l}\text { Macroergonomic } \\
\text { Analysis and Design } \\
\text { (MEAD) }\end{array}$ & $\begin{array}{l}\text { Combina subsistema tecnológico, pessoal e ambiente } \\
\text { externo a estrutura do sistema de trabalho da organização. }\end{array}$ \\
\hline
\end{tabular}

Fonte: Adaptado com base nos autores do quadro.

Dessa forma, é possível constatar que a ergonomia é uma disciplina científica que estuda as intervenções entre os seres humanos e outros elementos do sistema, e aplica teorias, princípios, dados e métodos a projetos que busquem otimizar o bem estar humano e o desempenho global de sistemas. Esse estudo envolve não apenas o ambiente físico, mas também os aspectos organizacionais e cognitivos. Além disso, atualmente, a ergonomia é aplicada em praticamente todos os tipos de atividades humanas independentemente do setor em que estas estejam inseridas. Neste contexto está à ergonomia organizacional ou macroergonomia, uma subdisciplina da ergonomia, que visa à otimização dos sistemas sócio-técnicos, incluindo as estruturas organizacionais, políticas ou regras, e processos, garantindo um ótimo funcionamento e efetividade do sistema. O projeto da macroergonomia pode ser analisado e desenhado de três formas descendente, ascendente e radial. Além disso, tanto a ergonomia como a ergonomia organizacional (ou macroergonomia) constroem seus conhecimentos a partir de observações e experimentações pela mensuração dos fenômenos, com a utilização de MPE's formado por um conjunto de técnicas. Dentre os tipos de MPE's possuem os participativos (o pesquisador passa a fazer parte ativa da solução do problema, podendo haver a inclusão de usuários ou consumidores no processo) e os convencionais (o pesquisador não interfere e não participa da situação observada). Os autores Wilson (1995), lida (2005), Stanton et al. (2005), Hendrick e Kleiner (2006), e Thiollent (2011) mencionam os métodos que podem ser utilizados na ergonomia organizacional. A próxima seção aborda os procedimentos metodológicos informando como esta pesquisa foi realizada. 


\section{Procedimentos metodológicos}

Esta seção visa apresentar os procedimentos metodológicos utilizados, bem como as etapas de pesquisa que se dividem em: 1) Fazer levantamento bibliográfico sobre ergonomia, ergonomia organizacional e MPE's para compor a fundamentação teórica; 2) Fazer revisão de literatura tradicional em quatro bases de dados eletrônicos, coletando artigos com ano de publicação de 2014 a 2016 e que abordam métodos de ergonomia e de ergonomia organizacional; 3 ) Ler e selecionar os artigos que mencionam a temática estudada; 4) Descrever os resultados referentes aos métodos que podem ser aplicados na ergonomia organizacional.

A revisão de literatura tradicional (CORDEIRO, 2007) foi realizada em junho de 2017, nas bases de dados eletrônicos: Revista Ação Ergonômica; 15 Congresso Internacional de Ergonomia e Usabilidade de Interfaces Humano-tecnologia (15\% ERGODESIGN); 1 을 Congresso Internacional de Ergonomia Aplicada (10 CONARG); e 11th International Symposium on Human Factors in Organisational Design and Management (11th ODAM). Essas bases de dados foram selecionadas por possuírem número maior de artigos científicos voltados para a área de ergonomia organizacional que é o foco da pesquisa. Por esse artigo ser o início de uma pesquisa optou-se, neste momento, pela leitura de 46 artigos coletados na base de dados do simpósio internacional 11th ODAM, devido ao fato de ser um dos principais eventos sobre o assunto. A figura 1 apresenta as informações da revisão de literatura tradicional de forma mais detalhada.

Figura 1. Especificações da Revisão de Literatura Tradicional

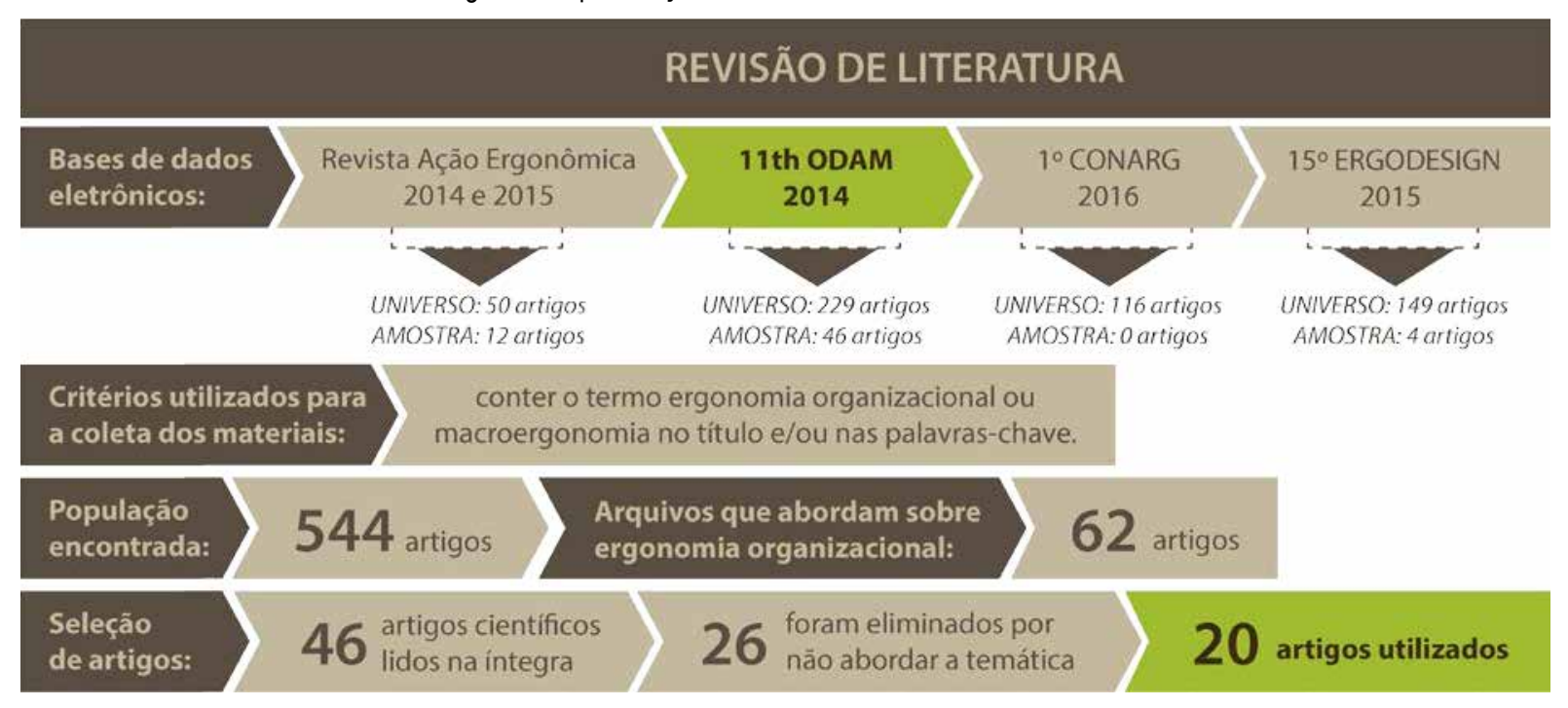

Fonte: Elaborado pelos autores.

\section{Métodos de pesquisa ergonômica}

Esta seção está dividida em dois itens: o primeiro constituído por um quadro que apresenta os MPE's direcionados para os aspectos organizacionais, encontrados na revisão de literatura; e o segundo retoma pontos importantes da fundamentação teórica relacionando-os com o resultado encontrado na revisão de literatura. 


\subsection{Métodos de ergonomia organizacional e suas aplicações}

O quadro 2 apresenta 20 artigos referentes a ergonomia organizacional que mostram diferentes tipos de MPE's que foram utilizados para compreender os aspectos organizacionais.

Quadro 2. Métodos encontrados nos artigos científicos

\begin{tabular}{|c|c|c|}
\hline Autor: & Método: & Aplicação: \\
\hline Heller-ono (2014) & $\begin{array}{l}\text { WIEP (Worksite } \\
\text { International Ergonomics } \\
\text { Process) }\end{array}$ & $\begin{array}{l}\text { É um modelo de macroergonomia de Hedrick, } \\
\text { centrado na concepção do sistema de trabalho } \\
\text { global, com a finalidade de promover eficiência e } \\
\text { eficácia dentro de uma organização. É um } \\
\text { processo organizacional enxuto, pró-ativo, } \\
\text { integrado e participativo, projetado para prevenir e } \\
\text { gerenciar lesões de trabalho auxiliando na gestão } \\
\text { da saúde e bem-estar dos funcionários. }\end{array}$ \\
\hline $\begin{array}{l}\text { Dellve, } \\
\text { Andreasson e } \\
\text { Jutengren (2014) }\end{array}$ & Questionário & $\begin{array}{l}\text { Aplicado na exploração de pré-requisitos } \\
\text { organizacionais para os gestores do setor público } \\
\text { sueco. }\end{array}$ \\
\hline Bartz et al. (2014) & $\begin{array}{l}\text { Entrevista (combinada com } \\
\text { pesquisas regulares, } \\
\text { análise de dados da } \\
\text { empresa, workshops) }\end{array}$ & $\begin{array}{l}\text { Para compreender o impacto da transformação do } \\
\text { trabalho e o processo de transformação na } \\
\text { organização, equipes e indivíduos. }\end{array}$ \\
\hline $\begin{array}{l}\text { Murphy et al. } \\
(2014)\end{array}$ & Grupo de foco & $\begin{array}{l}\text { Utilizado para coletar dados dos trabalhadores a } \\
\text { respeito de sua compreensão da segurança na } \\
\text { indústria de construção. }\end{array}$ \\
\hline $\begin{array}{l}\text { Goldberg e } \\
\text { Young-Corbett } \\
\text { (2014) }\end{array}$ & Entrevista & $\begin{array}{l}\text { Para compreender o processo de tomada de } \\
\text { decisões de especificação de materiais na } \\
\text { construção comercial. }\end{array}$ \\
\hline Syvänen (2014) & Pesquisa-ação participativa & $\begin{array}{l}\text { Com a finalidade de desenvolver a participação } \\
\text { em organizações de assistência social e de saúde } \\
\text { que prestam serviços para idosos. }\end{array}$ \\
\hline $\begin{array}{l}\text { Holden et al. } \\
(2014 a)\end{array}$ & $\begin{array}{l}\text { Caso 1: Estudo } \\
\text { experimental } \\
\text { Caso 2: Modelo de sistema } \\
\text { de trabalho } \\
\text { macroergonômico SEIPS } \\
\text { (Systems Engineering } \\
\text { Initiative for Patient Safety) }\end{array}$ & $\begin{array}{l}\text { Caso 1: Para avaliar o impacto de uma } \\
\text { intervenção macroergonômica sobre a saúde e o } \\
\text { desempenho do trabalhador do conhecimento. } \\
\text { Caso 2: Para o projeto e implementação da } \\
\text { tecnologia de bomba de infusão Smart em um } \\
\text { hospital. }\end{array}$ \\
\hline $\begin{array}{l}\text { Helali e } \\
\text { Abdollahpour } \\
\text { (2014) }\end{array}$ & $\begin{array}{l}\text { Método de fase pré- } \\
\text { macroergonômico (Phase } \\
\text { Method Pre- } \\
\text { Macroergonomics) }\end{array}$ & $\begin{array}{l}\text { Utilizado no processo de intervenção pré-macro- } \\
\text { ergonômico para despertar a necessidade de } \\
\text { mudança, melhorando o "sistema de condição de } \\
\text { trabalho" (saúde, segurança e ergonomia). Este } \\
\text { método foi implementado em uma usina fabril com } \\
\text { o objetivo de criar conscientização } \\
\text { macroergonômica na empresa, a fim de enfatizar } \\
\text { a importância de melhorar "sistemas de condições } \\
\text { de trabalho" (saúde, segurança e ergonomia). }\end{array}$ \\
\hline $\begin{array}{l}\text { Christina et al. } \\
(2014)\end{array}$ & Entrevista e grupo focal & $\begin{array}{l}\text { Com a finalidade de examinar como as } \\
\text { tecnologias e estratégias relacionadas à energia } \\
\text { são incorporadas à vida organizacional, por meio } \\
\text { de um estudo sobre a eficiência energética } \\
\text { comportamental dentro de um dos principais } \\
\text { varejistas do Reino Unido. }\end{array}$ \\
\hline
\end{tabular}

(continua) 


\begin{tabular}{|c|c|c|}
\hline & & (continuação) \\
\hline $\begin{array}{l}\text { Paterson et al. } \\
(2014)\end{array}$ & $\begin{array}{l}\text { Grupo focal e entrevista } \\
\text { semiestruturada }\end{array}$ & $\begin{array}{l}\text { Para testar as lições desenvolvidas a partir de } \\
\text { pesquisas anteriores na indústria mineira } \\
\text { australiana, referentes as } 10 \text { regras de platina, } \\
\text { como um quadro de ação para intervenções de } \\
\text { melhorias na cultura organizacional e gestão do } \\
\text { manejo da indústria ferroviária australiana. }\end{array}$ \\
\hline $\begin{array}{l}\text { Tavares, } \\
\text { Lourenço e } \\
\text { Coelho }(2014)\end{array}$ & $\begin{array}{l}\text { Questionário Psychococial } \\
\text { de Copenhaga (CoPsoQ) }\end{array}$ & $\begin{array}{l}\text { Utilizado para uma avaliação psicossocial } \\
\text { voluntária de trabalhadores de escritório em uma } \\
\text { companhia de serviços públicos, sob uma } \\
\text { perspectiva macroergonômica que articula } \\
\text { relações complexas em níveis organizacionais e } \\
\text { socio-econômicos que podem fornecer uma } \\
\text { explicação preliminar e exploratória das } \\
\text { mudanças na avaliação psicossocial. Este método } \\
\text { ajuda a examinar as demandas psicológicas do } \\
\text { trabalho, controle de trabalhadores sobre o } \\
\text { trabalho, a insegurança para o futuro } \\
\text { (preocupações em termos de perda de emprego } \\
\text { ou mudanças indesejáveis em condições de } \\
\text { trabalho), apoio social e qualidade de liderança, } \\
\text { demandas conflitantes, e a estima (em relação ao } \\
\text { respeito, recompensas e justiça vivenciadas em } \\
\text { troca do trabalho). }\end{array}$ \\
\hline $\begin{array}{l}\text { Holden et al. } \\
(2014 b)\end{array}$ & $\begin{array}{l}\text { Caso 1: Entrevista e } \\
\text { observação } \\
\text { Caso 2: Entrevista e grupo } \\
\text { de foco }\end{array}$ & $\begin{array}{l}\text { Este estudo considera os desafios relacionados } \\
\text { com a concepção e implementação da pesquisa } \\
\text { macroergonômica com os trabalhadores, pagos e } \\
\text { não pagos, menos estruturados ou marginalizados } \\
\text { pela sociedade. Foi traçado introspecções de dois } \\
\text { estudos recentes de pacientes cronicamente } \\
\text { doentes nas comunidades urbanas e rurais dos } \\
\text { US. }\end{array}$ \\
\hline $\begin{array}{l}\text { Osvalder e Alm } \\
(2014)\end{array}$ & Entrevista em profundidade & $\begin{array}{l}\text { Utilizado para identificar os desafios } \\
\text { organizacionais em projetos de redesenho no } \\
\text { domínio nuclear. }\end{array}$ \\
\hline $\begin{array}{l}\text { Conceição et al. } \\
\text { (2014) }\end{array}$ & Ergonomia participativa & $\begin{array}{l}\text { Este método foi desenvolvido e testado em } \\
\text { projetos de design de engenharia (uma linha de } \\
\text { empacotamento nova a fim de aumentar a } \\
\text { capacidade de produção) em um departamento de } \\
\text { uma fábrica de queijo. }\end{array}$ \\
\hline $\begin{array}{l}\text { Dul, Scheffers e } \\
\text { Stienstra (2014) }\end{array}$ & Questionário & $\begin{array}{l}\text { Desenvolveram um questionário padronizado para } \\
\text { medir três dimensões de desempenho (de tarefas, } \\
\text { contexto, e comportamento contraproducente) e } \\
\text { três dimensões de comportamento inovador } \\
\text { (geração, promoção e implementação de ideias). } \\
\text { O método pode ser usado em pesquisas } \\
\text { ergonômicas para medir o desempenho } \\
\text { autopercebido e o comportamento inovador, além } \\
\text { de poder ser aplicado para explorar as ligações } \\
\text { entre vários fatores do ambiente de trabalho } \\
\text { organizacional (por exemplo, a complexidade do } \\
\text { trabalho, autonomia). }\end{array}$ \\
\hline $\begin{array}{l}\text { Kelly e Riedel } \\
(2014)\end{array}$ & $\begin{array}{l}\text { Ergonomia participativa } \\
\text { combinada com o LSP } \\
\text { (Lego Serious Play) }\end{array}$ & $\begin{array}{l}\text { A combinação de métodos foi utilizada para } \\
\text { auxiliar equipes de trabalho em diferentes } \\
\text { aspectos organizacionais como na tomada de } \\
\text { decisão e processo de resolução de problemas. }\end{array}$ \\
\hline Sørensen (2014) & Questionário & $\begin{array}{l}\text { A pesquisa ergonômica precisa projetar o nível } \\
\text { organizacional e as intervenções. Para avaliar o } \\
\text { impacto de intervenções organizacionais (de }\end{array}$ \\
\hline
\end{tabular}

(continua) 


\begin{tabular}{|c|c|c|}
\hline & & $\begin{array}{l}\text { saúdeo cupacional que contribuem para melhorar } \\
\text { o desempenho organizacional) na satisfação do } \\
\text { usuário, do funcionário e na qualidade do serviço. }\end{array}$ \\
\hline $\begin{array}{l}\text { Eklund et al. } \\
(2014)\end{array}$ & Entrevista e questionárioP & $\begin{array}{l}\text { ara acompanhar e avaliar a implementaçãod a } \\
\text { produção enxuta em quinze unidades dentro de } \\
\text { sete organizações. Além de identificar fatores que } \\
\text { apoiam ou neutralizam a sustentabilidade e o } \\
\text { desenvolvimento de implementações de } \\
\text { produções enxutas em organizações públicas. }\end{array}$ \\
\hline $\begin{array}{l}\text { Robertson et al. } \\
(2014)\end{array}$ & $\begin{array}{l}\text { Ergonomia participativa e } \\
\text { IDEAS (Intervention Design } \\
\text { and Analyses Scorecard) }\end{array}$ & $\begin{array}{l}\text { O IDEAS foi desenvolvido e testado dentro do } \\
\text { método de ergonomia participativa para engajar } \\
\text { os funcionários da linha de frente na concepção } \\
\text { de intervenções integradas de segurança e saúde. }\end{array}$ \\
\hline $\begin{array}{l}\text { Poulsen, Ipsen e } \\
\text { Gish (2014) }\end{array}$ & $\begin{array}{l}\text { PoWRS program } \\
\text { (Prevention of Work } \\
\text { Related Stress) }\end{array}$ & $\begin{array}{l}\text { É um método participativo de intervenção } \\
\text { organizacional primária utilizado para prevenção } \\
\text { do estresse relacionado ao trabalho. Visa criar } \\
\text { mudanças que tenham um efeito positivo na } \\
\text { produtividade e no bem-estar. Além de testar se } \\
\text { os funcionários sem treinamento facilitador } \\
\text { poderiam ser nomeados como facilitadores } \\
\text { internos e tornarem-se líderes no processo. }\end{array}$ \\
\hline
\end{tabular}

Fonte: Adaptado com base nos autores do quadro.

Destaca-se o mapeamento por entrevista como o método mais utilizado pelos artigos científicos coletados. No entanto, o mapeamento por questionário organizacional, grupo de foco e a ergonomia participativa também se enquadram nos métodos mais utilizados na ordem em que foram mencionados (vide figura 2). Ressalta-se, ainda, que existe uma variedade de métodos utilizados uma vez que oito artigos mencionam o uso de métodos diferentes entre si.

Figura 2. Síntese dos métodos identificados
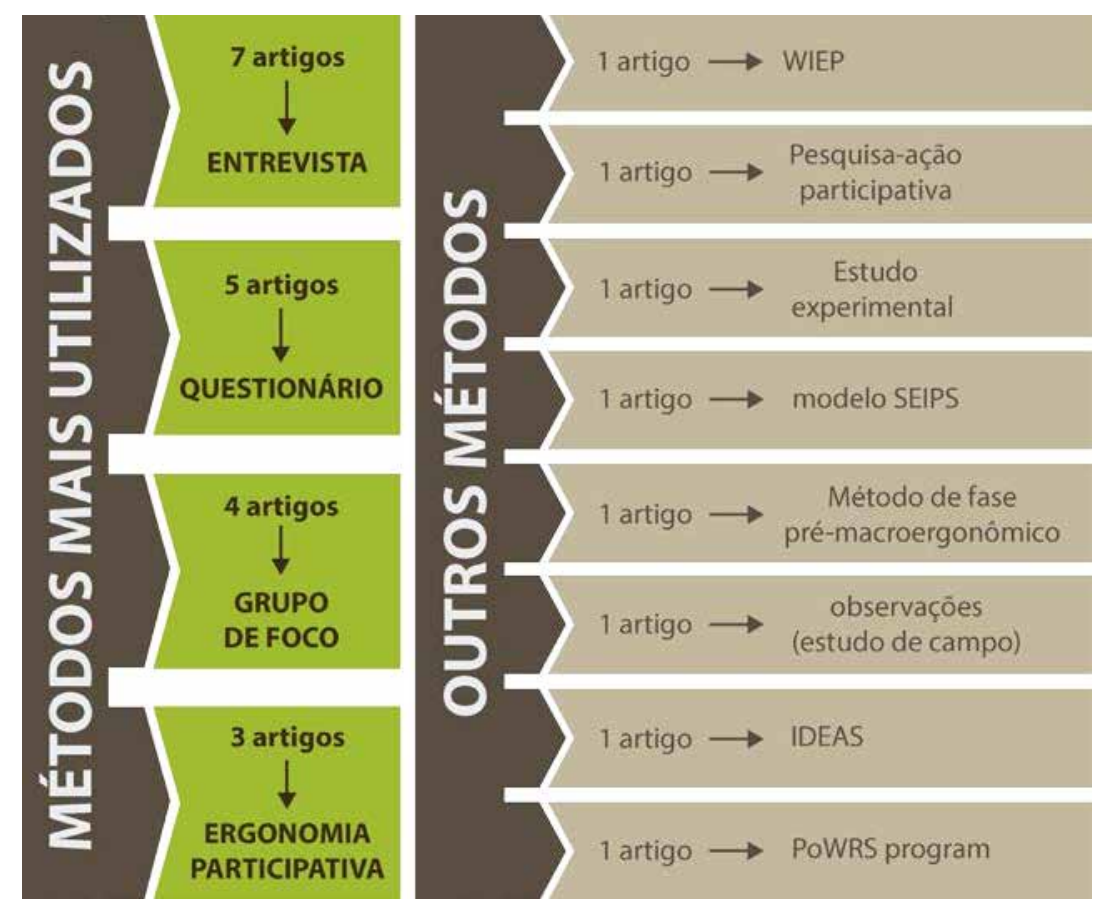

Fonte: Elaborado pelos autores. 
Diante do exposto e tendo como base a fundamentação teórica, apresenta-se na próxima seção a relação dos métodos, identificados nos artigos científicos, com os MPE's mencionados pelos autores citados na fundamentação teórica.

\subsection{Discussão dos resultados}

Como relatado na fundamentação teórica existem os métodos participativos e os convencionais. No quadro 1 e 2 é possível perceber que a pesquisa-ação e a ergonomia participativa são exemplos de métodos participativos, uma vez que envolvem a participação de pesquisadores, usuários e funcionários, e geralmente são mais utilizados na tomada de decisão, resolução de problemas e no projeto de treinamento de funcionários como confirmam Wilson (1995), lida (2005), Hendrick e Kleiner (2006), e Thiollent (2011). Além disso, os métodos WIEP (Worksite International Ergonomics Process), PoWRS (Prevention of Work Related Stress) program e IDEAS (Intervention Design and Analyses Scorecard), apresentados no quadro 2, são considerados participativos pelos autores Poulsen, Ipsen e Gish (2014), Robertson et al. (2014) e Heller-ono (2014).

Já o estudo de campo, experiência de laboratório, experiência de campo, mapeamento por questionário organizacional, mapeamento por entrevista, grupo de foco, método de fase pré-macroergonômico (Phase Method Pre-Macroergonomics) e modelo SEIPS (Systems Engineering Initiative for Patient Safety) são exemplos de métodos convencionais, utilizados em vários tipos de estudos organizacionais (IIDA, 2005; HENDRICK; KLEINER, 2006; HELALI; ABDOLLAHPOUR, 2014; HOLDEN et al., 2014a; HOLDEN et al., 2014b). Stanton et al. (2005) informa, na fundamentação teórica, outros métodos utilizados na ergonomia organizacional e que também são considerados métodos convencionais, como o: Cognitive Walk-Through Method (CWM); Kansei Engineering; HITOP Analysis; TOP-Modeler; CIMOP System; Anthropotechnology; Systems Analysis Tool (SAT); Macroergonomic Analysis of Structure (MAS); Macroergonomic Analysis and Design (MEAD).

Identificaram-se algumas similaridades, mostradas na figura 3, entre os métodos mencionados pelos autores da revisão de literatura (RV) e os citados pelos autores da fundamentação teórica (FT).

Figura 3. Similaridades identificadas nos métodos de ergonomia organizacional entre a RV e FT

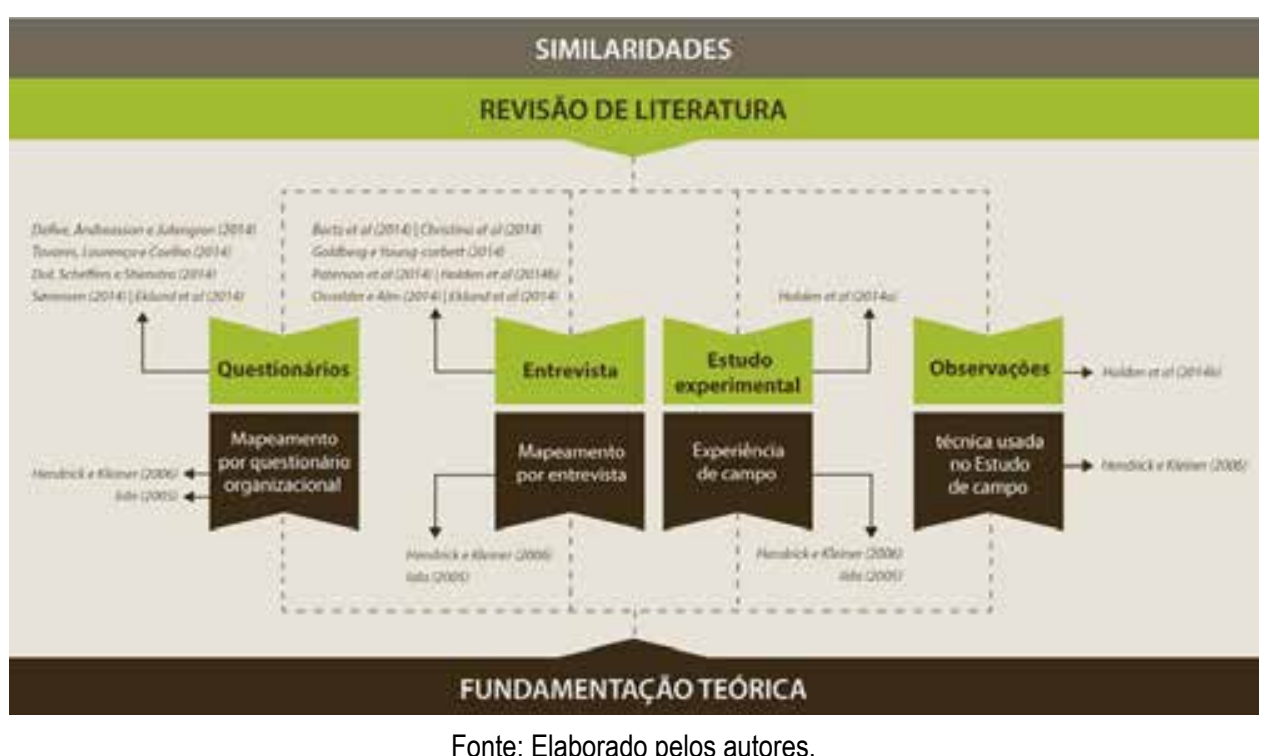

Fonte: Elaborado pelos autores. 
Percebe-se ainda que, como informado anteriormente, o mapeamento por entrevista é o método mais utilizado em sete artigos científicos coletados, seguido pelo questionário (cinco artigos), grupo de foco (quatro artigos) e ergonomia participativa (três artigos). As pesquisas dos autores Goldberg e Young-Corbett (2014) e dos Osvalder e Alm (2014) utilizam somente o mapeamento por entrevista. Já os demais artigos utilizam esse método combinado com outros, dentre estas pesquisas destaca-se a de: Bartz et al. (2014) que combina pesquisas regulares, análise de dados da empresa, workshops e entrevistas pessoais; Christina et al. (2014), Paterson et al. (2014) e Holden et al. (2014b) ao mesclarem entrevistas e grupos focais, mas este último autor apresenta outro caso em que intercala entrevistas com observações; e Eklund et al. (2014) que utiliza entrevistas combinadas com questionários. Além disso, o Robertson et al. (2014) e Kelly e Riedel (2014) também utilizam métodos mistos. Robertson et al. (2014) utiliza a ergonomia participativa juntamente com o IDEAS (Intervention Design and Analyses Scorecard), e Kelly e Riedel (2014) mescla a ergonomia participativa com o LSP (Lego Serious Play). Neste contexto, Hendrick e Kleiner (2006) informam que a experiência de campo é frequentemente combinada com uma abordagem de ergonomia participativa, já que aumenta a probabilidade dos empregados e suporte gerencial aceitarem e apoiarem as mudanças causadas pela intervenção ergonômica, isto confirma que é possível aplicar métodos mistos quando for necessário.

Os autores lida (2005), Hendrick e Kleiner (2006), Falzon (2007), Másculo e Vidal (2011) informaram, na fundamentação teórica, que a ergonomia organizacional compreende: as comunicações; a concepção do trabalho; a gestão dos coletivos; a concepção dos horários de trabalho; programação do trabalho em grupo; ergonomia comunitária; as novas formas de trabalho; projeto participativo; trabalho cooperativo; cultura organizacional; organizações em rede; teletrabalho; e gestão da qualidade. Além disso, também estão inclusos os aspectos relacionados com a produtividade, qualidade, tomada de decisão, saúde e segurança do empregado, fatores psicossociais de conforto, motivação intrínseca, compromisso e percepção da qualidade de vida no trabalho. O quadro 2 mostra que os MPE's organizacionais são utilizados para compreender ou melhorar estes aspectos dentro das organizações (figura 4).

Figura 4. MPE's direcionados para os aspectos organizacionais.

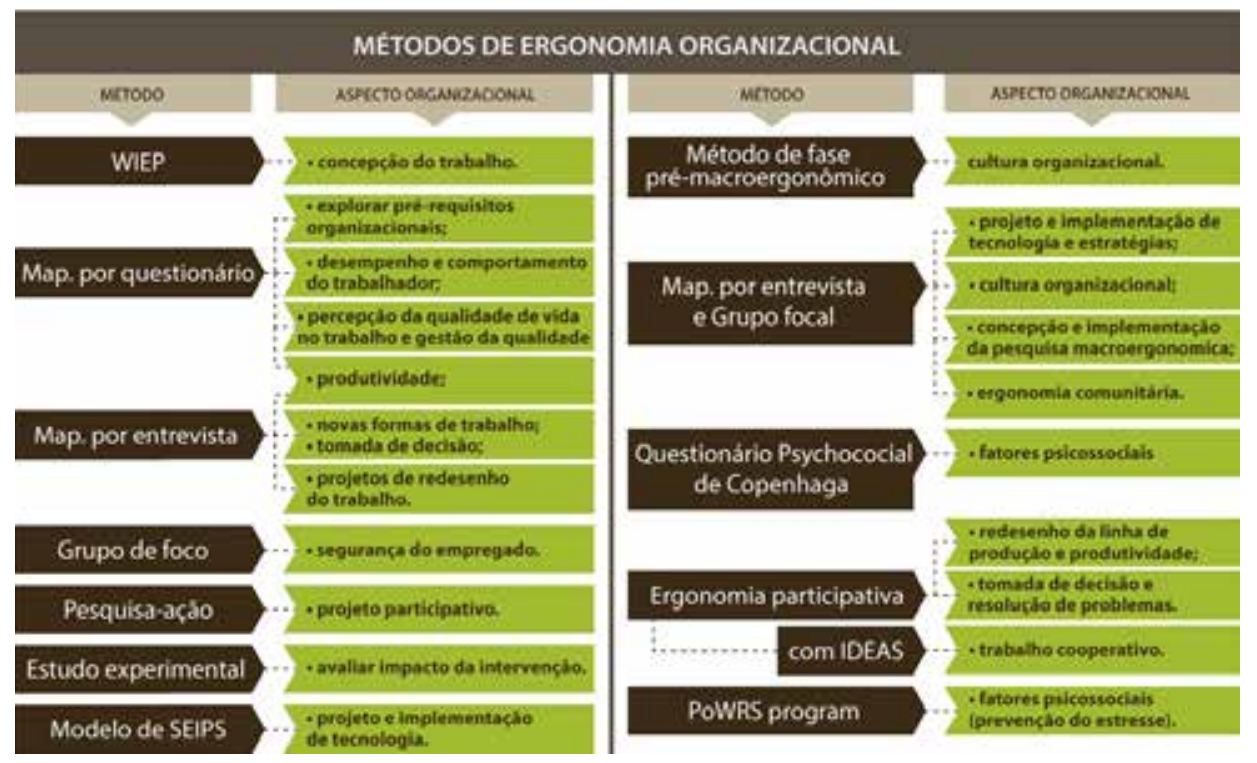

Fonte: Elaborado pelos autores. 
Dessa forma, percebe-se que existe uma grande variedade de métodos voltados para a ergonomia organizacional que auxiliam a identificar e compreender os aspectos organizacionais, e dentre esta variedade de MPE's os mais utilizados são o mapeamento por entrevista, mapeamento por questionário, grupo de foco e ergonomia participativa. A próxima seção relata as conclusões identificadas nesta pesquisa.

\section{Conclusão}

Retomando os questionamentos feitos na pergunta de pesquisa: Quais são os métodos de ergonomia organizacional existentes? E para quais finalidades estes métodos têm sido utilizados?, e o objetivo geral estabelecido: identificar os métodos e técnicas utilizadas, preferencialmente no contexto de MPE's organizacional, conclui-se que os mesmos foram respondidos ao apresentar: no quadro 1 os MPE's utilizados na macroergonomia encontrados nos livros que compõem a fundamentação teórica; no quadro 2, disposto na seção cinco, os métodos aplicados por 20 artigos científicos, destacando a entrevista como o método mais utilizado em sete artigos científicos, bem como o questionário (cinco artigos), grupo de foco (quatro artigos) e a ergonomia participativa (três artigos) que também se enquadram nos métodos mais utilizados.

Ressaltou-se, ainda sobre o quadro 2, que possui uma variedade de métodos utilizados para a compreensão e aperfeiçoamento dos aspectos organizacionais, visto que oito artigos utilizaram métodos diferentes. Dentre estes estão os MPE's: WIEP (Worksite International Ergonomics Process); pesquisa-ação participativa; estudo experimental ou experiência de campo; modelo de sistema de trabalho macroergonômico SEIPS (Systems Engineering Initiative for Patient Safety); Método de fase pré-macroergonômico (Phase Method Pre-Macroergonomics); observação ou estudo de campo; IDEAS (Intervention Design and Analyses Scorecard); e PoWRS (Prevention of Work Related Stress) program.

É relevante evidenciar que existem os métodos participativos e os convencionais. Nessa pesquisa foi identificado que a: pesquisa-ação, ergonomia participativa, WIEP (Worksite International Ergonomics Process), PoWRS (Prevention of Work Related Stress) program e IDEAS (Intervention Design and Analyses Scorecard) são considerados métodos participativos; e o mapeamento por entrevista, mapeamento por questionário organizacional, grupo de foco, estudo experimental ou experiência de campo, modelo de sistema de trabalho macroergonômico SEIPS (Systems Engineering Initiative for Patient Safety), método de fase pré-macroergonômico (Phase Method Pre-Macroergonomics), observação ou estudo de campo são exemplos de métodos convencionais.

Também se conclui que os MPE's são utilizados para compreender e/ou melhorar a: comunicação; concepção do trabalho; gestão dos coletivos; concepção dos horários de trabalho; programação do trabalho em grupo; ergonomia comunitária; as novas formas de trabalho; projeto participativo; trabalho cooperativo; cultura organizacional; organizações em rede; teletrabalho; gestão da qualidade; e os aspectos relacionados com a produtividade, qualidade, tomada de decisão, saúde e segurança 
do empregado, fatores psicossociais de conforto, motivação intrínseca, compromisso e percepção da qualidade de vida no trabalho. Os procedimentos metodológicos adotados (levantamento bibliográfico; revisão de literatura tradicional; leitura e seleção de artigos; e descrição dos resultados) mostraram-se pertinentes para esta pesquisa inicial, pois contribuíram para a compreensão da ergonomia organizacional bem como dos MPE's que são aplicados para aperfeiçoar os aspectos organizacionais, ajudando a atingir os objetivos propostos e a responder a problemática encontrada.

Este artigo vem contribuir no meio acadêmico com um panorama de métodos ergonômicos que podem ser aplicados na ergonomia organizacional ou macroergonomia para diversas finalidades como foi informado anteriormente. Contribui, também, para o âmbito profissional, pois os ergonomistas podem utilizar esse panorama desenvolvido para verificar qual método adequa-se melhor na situação a ser estudada dentro de uma organização. Como limitação de pesquisa ressalta-se que embora tenham sido encontrados mais 16 artigos científicos, além dos mencionados, neste momento optou-se por abordar apenas os artigos do evento ODAM, por isso, sugere-se como continuação da pesquisa: a leitura desses 16 artigos científicos e o desenvolvimento de outro artigo abordando o conteúdo destes; além da ampliação da pesquisa, realizando uma revisão sistemática ou bibliométrica acrescentando outras bases de dados.

\section{REFERÊNCIAS}

ABRAHÃO, Júlia; SZNELWAR, Laerte Idal; SILVINO, Alexandre; SARMET, Maurício; PINHO, Diana. Introdução à ergonomia: da prática à teoria. 1. ed. São Paulo: Blucher, 2009.

BARTZ, Michael; HARTNER-TIEFENTHALER, Martina; WÄRZNER, Alessandro; KÖSZEGI, Sabine T. Case study on an accelerated approach to a new world of work transformation. In: 11th INTERNATIONAL SYMPOSIUM ODAM, 2014. Anais do 11th International Symposium on Human Factors in Organisational Design and Management, Copenhagen, p. 535-536.

CHRISTINA, Sian; WATERSON, Patrick; DAINTY, Andrew; DANIELS, Kevin. Improving energy efficiency in the retail sector through job redesign: a sociotechnical systems approach. In: 11th INTERNATIONAL SYMPOSIUM ODAM, 2014. Anais do 11th International Symposium on Human Factors in Organisational Design and Management, Copenhagen, p. 177-181.

CONCEIÇÃO, Carolina; BROBERG, Ole; ALDRICH, Per T.; BANKE, Palle. Participatory ergonomics in industrial engineering projects: The case of a new cheese packaging line work system. In: 11th INTERNATIONAL SYMPOSIUM ODAM, 2014. Anais do 11th International Symposium on Human Factors in Organisational Design and Management, Copenhagen, p. 1071-1072. 
CORRÊA, Vanderlei Moraes; BOLETTI, Rosane Rosner. Ergonomia: fundamentos e aplicações. Porto Alegre: Bookman, 2015.

CRESWELL, J. W. Projeto de pesquisa: métodos qualitativo, quantitativo e misto. Porto Alegre: SAGE, 2010.

DUL, Jan; SCHEFFERS, Martijn; STIENSTRA, Marten. Performance and innovative behavior of employees. Development and application of a measurement tool for workplace research. In: 11th INTERNATIONAL SYMPOSIUM ODAM, 2014. Anais do 11th International Symposium on Human Factors in Organisational Design and Management, Copenhagen, p. 313.

DELLVE, Lotta; ANDREASSON, J; JUTENGREN, G. A prospective study of the importance of leadership support for healthrelated sustainability and participatory approaches towards employees. In: 11th INTERNATIONAL SYMPOSIUM ODAM, 2014. Anais do 11th International Symposium on Human Factors in Organisational Design and Management, Copenhagen, p. 1039.

EKLUND, Jörgen; HALVARSSON, Agneta; KOCK, Henrik; LINDSKOG, Pernilla; SVENSSON, Lennart. Sustainability and development of lean implementations. In: 11th INTERNATIONAL SYMPOSIUM ODAM, 2014. Anais do 11th International Symposium on Human Factors in Organisational Design and Management, Copenhagen, p. 165-169.

FALZON, Pierre. Ergonomia. São Paulo: Blucher, 2007.

GIL, Antonio Carlos. Como elaborar projetos de pesquisa. São Paulo: Atlas, 2010.

GOLDBERG, Ari; YOUNG-CORBETT, Deborah. Decision making in commercial construction. 2014. In: 11th INTERNATIONAL SYMPOSIUM ODAM, 2014. Anais do 11th International Symposium on Human Factors in Organisational Design and Management, Copenhagen, p. 907-910.

GUÉRIN, F.; LAVILLE, A.; DANIELLOU, F.; DURAFFOURG, J.; KERGUELEN, A. Compreender o trabalho para transformá-lo: a prática da ergonomia. São Paulo: Edgard Blucher, 2001.

HELALI, Faramarz; ABDOLLAHPOUR, Nosrat. How could you implement 'awakened need of change' for the applying ergonomics to work system in industrially developing countries? In: 11th INTERNATIONAL SYMPOSIUM ODAM, 2014. Anais do 11th International Symposium on Human Factors in Organisational Design and Management, Copenhagen, p. 251-257.

HELLER-ONO, Alison. A prospective study of a macroergonomics process over five years demonstrates significant prevention of workers' compensation claims resulting in projected savings. In: 11th INTERNATIONAL SYMPOSIUM ODAM, 2014. Anais do 
11th International Symposium on Human Factors in Organisational Design and Management, Copenhagen, p. 261-266.

HENDRICK, Hal W. Future directions in macroergonomics. Ergonomics, v. 38, abr. 1995, p. 1617-1624.

HENDRICK, Hal W.; KLEINER, Brian M. Macroergonomia: uma introdução aos projetos de sistemas de trabalho. Rio de Janeiro: Virtual Científica, 2006.

HOLDEN, Richard J.; ROBERTSON, Michelle M.; NEUMANN, Patrick; ZINK, Klaus J.; CARAYON, Pascale. Documenting and sharing macroergonomic success stories: nearly two decades of good ergonomics. In: 11th INTERNATIONAL SYMPOSIUM ODAM, 2014a. Anais do 11th International Symposium on Human Factors in Organisational Design and Management, Copenhagen, p. 259-260.

HOLDEN, Richard J.; McDOUGALD SCOTT, Amanda M.; CARAYON, Pascale; HOONAKKER, Peter L. T.; HUNDT, Ann S.. Macroergonomics in the wild: research challenges in community settings. In: 11th INTERNATIONAL SYMPOSIUM ODAM, 2014b. Anais do 11th International Symposium on Human Factors in Organisational Design and Management, Copenhagen, p. 613-614.

IIDA, Itiro. Ergonomia: projeto e produção. 2. ed. São Paulo: Edgard Blücher, 2005.

IEA, International Ergonomics Association. Definition and domains of ergonomics. 2000. Disponível em: http://www.iea.cc/whats/index.html. Acesso em: 23 mai. 2017.

KELLY, Martina; RIEDEL, Ralph. Serious play approaches in participative ergonomics. In: 11th INTERNATIONAL SYMPOSIUM ODAM, 2014. Anais do 11th International Symposium on Human Factors in Organisational Design and Management, Copenhagen, p. 717-722.

MAGGI, Bruno. Do agir organizacional: um ponto de vista sobre o trabalho, o bem estar, a aprendizagem. São Paulo: Edgard Blücher, 2006.

MÁSCULO, Francisco Soares; VIDAL, Mario Cesar. Ergonomia: trabalho adequado e eficiente. Rio de Janeiro: Elsevier, 2011.

MERINO, Eugenio Andrés Díaz. Fundamentos da ergonomia. Apostila. Florianópolis: UFSC, 2011. Disponível em: https://moodle.ufsc.br/pluginfile.php/2034406/mod_resource/content/1/Ergo_Fundamentos.pdf. Acesso em: 24 mar. 2017.

MURPHY, Lauren A.; DENNERLEIN, Jack T.; ROBERTSON, Michelle M.; CABAN-MARTINEZ, Alberto J. Conceptualizing safety climate in the U.S. construction industry. In: 11th INTERNATIONAL SYMPOSIUM ODAM, 2014. Anais do 11th International Symposium on Human Factors in Organisational Design and Management, Copenhagen, p. 
MURREL, K. F. H. Ergonomics: man and his working environment. London: Chapman and Hall, 1965.

OSVALDER, Anna-Lisa; ALM, Håkan. Organisational challenges associated with design and implementation of technology in re-design projects in the nuclear domain. In: 11th INTERNATIONAL SYMPOSIUM ODAM, 2014. Anais do 11th International Symposium on Human Factors in Organisational Design and Management, Copenhagen, p. 739-744.

PATERSON, Jessica L; BLEWETT, Verna; RAINBIRD, Sophia; ETHERTON, Hayley. Keeping rail on track: good practice work health and safety in the Australian rail industry. In: 11th INTERNATIONAL SYMPOSIUM ODAM, 2014. Anais do 11th International Symposium on Human Factors in Organisational Design and Management, Copenhagen, p. 441-446.

POULSEN, Signe; IPSEN, Christine; GISH, Liv. The use of the Chronicle Workshop as a method for evaluating participatory interventions. In: 11th INTERNATIONAL SYMPOSIUM ODAM, 2014. Anais do 11th International Symposium on Human Factors in Organisational Design and Management, Copenhagen, p. 725-730.

ROBERTSON, Michelle M.; HENNING, Robert; WARREN, Nicholas; NOBREGA, Suzanne; DOVE-STEINKAMP, Megan; BIZARRO, Andrea. The intervention design and analysis scorecard: a business decision-making tool for participatory design of integrated health and safety interventions in the workplace. In: 11th INTERNATIONAL SYMPOSIUM ODAM, 2014. Anais do 11th International Symposium on Human Factors in Organisational Design and Management, Copenhagen, p. 611-612.

SØRENSEN, Ole Henning. Service quality as goal and outcome of ergonomics research: user and employee perspectives. In: 11th INTERNATIONAL SYMPOSIUM ODAM, 2014. Anais do 11th International Symposium on Human Factors in Organisational Design and Management, Copenhagen, p. 317-322.

STANTON, Neville Anthony; HEDGE, Alan; BROOKHUIS, Karel; SALAS, Eduardo; e HENDRICK, Hal W. Handbook of human factors and ergonomics methods. Boca Raton: CRC Press LLC, 2005.

SYVÄNEN, Sirpa. Dialogic leadership and participatory development: key factors of quality of working life and performance. In: 11th INTERNATIONAL SYMPOSIUM ODAM, 2014. Anais do 11th International Symposium on Human Factors in Organisational Design and Management, Copenhagen, p. 397-402.

TAVARES, Carla S. D.; LOURENÇO, Miguel L.; COELHO, Denis A.. Longitudinal psychosocial assessment of office workers in a utility company. In: 11th INTERNATIONAL 
SYMPOSIUM ODAM, 2014. Anais do 11th International Symposium on Human Factors in Organisational Design and Management, Copenhagen, p. 739-744.

THIOLLENT, Michel. Metodologia da pesquisa-ação. 18. ed. São Paulo: Cortez, 2011. VIRGILLITO, Salvatore Benito. Pesquisa de marketing: uma abordagem quantitativa e qualitativa. São Paulo: Saraiva, 2010.

WILSON, J. R. Ergonomics and participation. In: WILSON, J. R.; CORLETT, E. N. (Org.) Evaluation o human work: a practical ergonomics methodology. London: Taylor \& Francis, 1995, p.1071-1096.

WISNER, A. Por dentro do trabalho: ergonomia, método e técnica. São Paulo: FTD Oboré, 1987. 